\title{
Article
}

\section{Probing Many-Body Systems near Spectral Degeneracies}

\author{
Klaus Ziegler $\mathbb{D}$
}

Citation: Ziegler, K. Probing Many-Body Systems near Spectral Degeneracies. Symmetry 2021, 13, 1796. https://doi.org/10.3390/ sym13101796

Academic Editors: V. I. Yukalov, V. S. Bagnato and Rashid G. Nazmitdinov

Received: 30 August 2021

Accepted: 23 September 2021

Published: 26 September 2021

Publisher's Note: MDPI stays neutral with regard to jurisdictional claims in published maps and institutional affiliations.

Copyright: (C) 2021 by the author. Licensee MDPI, Basel, Switzerland. This article is an open access article distributed under the terms and conditions of the Creative Commons Attribution (CC BY) license (https:/ / creativecommons.org/licenses/by/ $4.0 /)$.
Institut für Physik, Universität Augsburg, D-86135 Augsburg, Germany; klaus.ziegler@physik.uni-augsburg.de

\begin{abstract}
The diagonal elements of the time correlation matrix are used to probe closed quantum systems that are measured at random times. This enables us to extract two distinct parts of the quantum evolution, a recurrent part and an exponentially decaying part. This separation is strongly affected when spectral degeneracies occur, for instance, in the presence of spontaneous symmetry breaking. Moreover, the slowest decay rate is determined by the smallest energy level spacing, and this decay rate diverges at the spectral degeneracies. Probing the quantum evolution with the diagonal elements of the time correlation matrix is discussed as a general concept and tested in the case of a bosonic Josephson junction. It reveals for the latter characteristic properties at the transition to Hilbert-space localization.
\end{abstract}

Keywords: dynamics of closed quantum systems; random probing; separation of time scales; Hilbertspace localization

\section{Introduction}

Symmetries play a central role in classical and in quantum many-body systems. They determine the macroscopic behavior of these systems. Moreover, symmetries of macroscopic states reflect symmetries and spontaneous symmetry breaking of the underlying system. For instance, the Hamiltonian of the Ising model has a global $Z_{2}$ symmetry, and with ferromagnetic nearest-neighbor spin-spin coupling its ground state is two-fold degenerate with $|\uparrow, \ldots, \uparrow\rangle$ and $|\downarrow, \ldots, \downarrow\rangle$. Spin flip dynamics will create excited states that are generically not $Z_{2}$ symmetric. Moreover, we can prepare the initial state of the Ising system in one of the ground states, e.g., in the state $|\uparrow, \ldots, \uparrow\rangle$ that breaks the $Z_{2}$ symmetry. Then the dynamics prefer the vicinity of the initial state because it would cost too much energy to overcome the barrier to the other ground state $|\downarrow, \ldots, \downarrow\rangle$, although the Hamiltonian and the spin-flip operator obey the $Z_{2}$ symmetry. As a result, the system experiences dynamical symmetry breaking by which it prefers to remain in the vicinity of a symmetry-broken state.

In the following we consider the unitary evolution of closed quantum many-body systems. It is based on the idea that the extraction of information about the quantum system in an experiment is limited. In other words, not all properties or degrees of freedom of the quantum model are accessible by an experiment. Typical exceptions are the return and transition probabilities for quantum states. Moreover, quantum systems have complex dynamics. Although the evolution is deterministic, even for a few particles it can looks erratic, similarly to a classical random walk. Such behavior suggests a statistical approach to extract generic information about the quantum evolution, using averaged quantities. A statistical approach is also supported by the fact that large sets of experimental data are available whose properties can be treated statistically. This idea is not new and found very successful realization in random matrix theory (RMT). It has been applied to many physical systems, such as nuclei, atoms and mesoscopic systems [1-7]. The motivation for RMT is that there is no way of knowing the Hamiltonian of even a relatively small manybody quantum system, such as an atomic nucleus. On the other hand, the spectra of these systems, complex though they are, have some characteristic features, such as level repulsion. Thus, instead of guessing a specific Hamiltonian, a random ensemble of Hamiltonians 
is chosen, which describes the generic features of a class of quantum systems. The class is characterized by the invariance of the random ensemble with respect to symmetry transformations. These are typically orthogonal, unitary or symplectic transformations. Another application of RMT has been recently proposed for the description of random measurements. It is based on Dyson's circular matrix ensemble $[3,6,8,9])$, which represents random unitary matrices and has been used as a tool to determine the trace of powers of the density matrix and the related Rényi entropy [10-14].

In contrast to these RMT approaches, we consider in the following a dynamical approach in which only the time of a measurement is random, whereas the energy levels $\left\{E_{j}\right\}$ of the Hamiltonian $H$ and the overlaps $\left\langle E_{j} \mid \Psi_{0}\right\rangle,\left\langle E_{j} \mid \Psi\right\rangle$ of the energy eigenstates $\left\{\left|E_{j}\right\rangle\right\}$ with a given initial state $\left|\Psi_{0}\right\rangle$ and a measured state $|\Psi\rangle$ are not random. This leads to the time correlation matrix (TCM) as the central tool for the definition of the statistical model, instead of the random ensemble of Hamiltonians in RMT. We employ this approach, which was previously described in [15] to analyze the evolution of the return and transition probabilities. In more concrete terms, for a given time $t_{k}$ we evaluate (in a calculation or in a real experiment) the probability $p_{k}$ that the system is in a certain state. Then we evaluate the probabilities $\left\{p_{1}, p_{2}, \ldots\right\}$ at different discrete and randomly chosen times $\left\{t_{1}, t_{2}, \ldots\right\}$. This can be translated into practical observations, for which it was assumed that each experiment was prepared in the same initial state and all measurements were performed for the same final state of the evolution at different times. These experiments provide an ensemble of probabilities $\left\{p_{1}, p_{2}, \ldots\right\}$ with the corresponding times $\left\{t_{1}, t_{2}, \ldots\right\}$.

For given overlaps $\left\langle E_{j} \mid \Psi_{0}\right\rangle,\left\langle E_{j} \mid \Psi\right\rangle$ we can immediately predict some restrictions for the evolution of the probability $p_{k}$ in the $N$-dimensional Hilbert space. When the overlaps vanish for some of the eigenstates $\left|E_{n}\right\rangle$, the evolution cannot reach those states and the accessible Hilbert space is restricted to the states $\left|E_{j}\right\rangle$ with $j \neq n$. This reduction of the Hilbert space can be interpreted as Hilbert-space localization [16] or Hilbert-space fragmentation [17]. This effect can be associated with spontaneous symmetry breaking, induced by the choice of the initial and measured states. In the case that the overlaps with some states are not strictly zero but very small, the access to those states may be negligible and can be ignored. This corresponds to complex dynamical behavior and requires a careful analysis. It is addressed briefly for the example of a bosonic Josephson junction in Section 3, where the mirror symmetry of the junction is spontaneously broken.

This paper is organized as follows. After the definition of the TCM in Section 2 we focus on the properties of its diagonal elements (Section 2.1). Then the effect of spectral degeneracies on the diagonal TCM elements are discussed in Section 2.2. In Section 3, we analyze the diagonal TCM elements in the specific example of a bosonic Josephson junction.

\section{Time Correlation Matrix}

We consider the transition amplitude from $\left|\Psi_{0}\right\rangle$ to $|\Psi\rangle$

$$
u_{k}=\left\langle\Psi\left|e^{-i H t_{k}}\right| \Psi_{0}\right\rangle,
$$

which is based on the unitary evolution with the Hamiltonian $H$ from the initial state $\left|\Psi_{0}\right\rangle$. The probability of measuring the state $|\psi\rangle$ at time $t_{k}$ is given by $\left|\left\langle\Psi\left|e^{-i H t_{k}}\right| \Psi_{0}\right\rangle\right|^{2}$. In other words, $p_{k}=\left|u_{k}\right|^{2}$ is the probability of finding the quantum system in the state $|\Psi\rangle$ after the unitary evolution from the initial state $\left|\Psi_{0}\right\rangle$ over the time $t_{k}$. Since the evolution is defined by the Hamiltonian $H$, we consider its eigenstates $\left\{\left|E_{j}\right\rangle\right\}_{j=1, \ldots, N}$ and its corresponding eigenvalues $\left\{E_{j}\right\}_{j=1, \ldots, N}$ and write the amplitude in spectral representation as

$$
u_{k}=\sum_{j=1}^{N}\left\langle\Psi \mid E_{j}\right\rangle\left\langle E_{j} \mid \Psi_{0}\right\rangle e^{-i E_{j} t_{k}} \equiv \sum_{j=1}^{N} q_{j} e^{-i E_{j} t_{k}} .
$$


Although the phases are not directly experimentally observable, their effects can be detected through the interference of different quantum states. For instance, the product of amplitudes at different times with probabilities of interfering amplitudes reads

$$
\begin{gathered}
u_{k}^{*} u_{k^{\prime}}+u_{k^{\prime}}^{*} u_{k}=\frac{1}{2}\left(\left|u_{k}+u_{k^{\prime}}\right|^{2}-\left|u_{k}-u_{k^{\prime}}\right|^{2}\right), \\
i\left(u_{k}^{*} u_{k^{\prime}}-u_{k^{\prime}}^{*} u_{k}\right)=\frac{1}{2}\left(\left|u_{k}+i u_{k^{\prime}}\right|^{2}-\left|u_{k}-i u_{k^{\prime}}\right|^{2}\right),
\end{gathered}
$$

where the probabilities can be detected in interferometric measurements. These relations suggest considering the correlation of the amplitudes $u_{k}$ and $u_{k^{\prime}}$ at different times through the TCM $\left\langle u_{k}^{*} u_{k^{\prime}}\right\rangle_{\tau}$, where the average $\langle\ldots\rangle_{\tau}$ is taken from the distribution of times $\left\{t_{k}\right\}$ as a result of inaccurate clocks: The time is measured by a clock in each laboratory by counting time steps $\left\{\tau_{n}\right\}$. These clocks have a limited accuracy, such that the time steps vary randomly. This implies a sequence of measurements in each laboratory, where the clocks indicate $k$ time steps and a total evolution time $t_{k}=\tau_{1}+\cdots+\tau_{k}$ for different values of $k$. Now we can compare the measured sequences of different laboratories. This provides a distribution of results for $u_{k}^{*} u_{k^{\prime}}$ due to different inaccurate clocks, where we assume that the fluctuations of the time steps $\left\{\tau_{n}\right\}$ are independently and equally distributed. Then the TCM is, as a spectral representation,

$$
\begin{gathered}
\left\langle u_{k}^{*} u_{k^{\prime}}\right\rangle_{\tau}=\sum_{j, j^{\prime}} q_{j}^{*} q_{j^{\prime}}\left\langle e^{i E_{j}\left(\tau_{1}+\cdots+\tau_{k}\right)} e^{-i E_{j^{\prime}}\left(\tau_{1}+\cdots+\tau_{k^{\prime}}\right)}\right\rangle_{\tau} \\
=\sum_{j, j^{\prime}} q_{j}^{*} q_{j^{\prime}} \begin{cases}\left\langle e^{i\left(E_{j}-E_{j^{\prime}}\right)\left(\tau_{1}+\cdots+\tau_{k}\right)}\right\rangle_{\tau}\left\langle e^{-i E_{j^{\prime}}\left(\tau_{k+1}+\cdots+\tau_{k^{\prime}}\right)}\right\rangle_{\tau} & k^{\prime}>k \\
\left\langle e^{i\left(E_{j}-E_{j^{\prime}}\right)\left(\tau_{1}+\cdots+\tau_{k^{\prime}}\right)}\right\rangle_{\tau}\left\langle e^{i E_{j^{\prime}}\left(\tau_{k^{\prime}+1}+\cdots+\tau_{k}\right)}\right\rangle_{\tau} & k^{\prime}<k . \\
\left\langle e^{i\left(E_{j}-E_{j^{\prime}}\right)\left(\tau_{1}+\cdots+\tau_{k}\right)}\right\rangle_{\tau} & k^{\prime}=k\end{cases}
\end{gathered}
$$

When $\lambda_{j}=\left\langle e^{i E_{j} \tau}\right\rangle_{\tau}$ and $\lambda_{j j^{\prime}}=\left\langle e^{i\left(E_{j}-E_{j^{\prime}}\right) \tau}\right\rangle_{\tau}$ the TCM elements become

$$
\left\langle u_{k}^{*} u_{k^{\prime}}\right\rangle_{\tau}=\sum_{j, j^{\prime}} q_{j}^{*} q_{j^{\prime}}\left\{\begin{array}{ll}
\lambda_{j j^{\prime}}^{k} \lambda_{j^{\prime}}^{k^{\prime}-k} & k^{\prime} \geq k \\
\lambda_{j j^{\prime}}^{k^{\prime}} \lambda_{j}^{* k-k^{\prime}} & k^{\prime}<k
\end{array} .\right.
$$

The TCM decays exponentially with $\left|k-k^{\prime}\right|$, provided that $\left|\lambda_{j}\right|<1$. Moreover, for fixed $\left|k-k^{\prime}\right|$ the TCM is constant for the diagonal elements $\lambda_{j j}=1$. This reflects the fact that a unitary evolution between the same energy eigenstates gives just a phase factor $e^{-i E_{j} \tau}$ (cf. Equation (2)). For different energy states, on the other hand, these phase factors lead to a decay due to interference effects after the time average.

\subsection{Diagonal Elements of the TCM}

The diagonal TCM element $\left\langle\left|u_{k}\right|^{2}\right\rangle_{\tau}$ is the probability of measuring the state $|\Psi\rangle$ at time $t_{k}$. Before time averaging, the expression $\left|u_{k}\right|^{2}$ is a diagonal element of the density matrix $\rho\left(t_{k}\right)$ with respect to the state $|\Psi\rangle$. The trace of $\left|u_{k}\right|^{2}$ with respect to all states $|\Psi\rangle$ of the underlying Hilbert space is the spectral form factor, often used for the characterization of many-body quantum chaos [18-20]. We only mention this, but do not study it here. In comparison with the spectral form factor, the diagonal elements of the TCM refer to a specific measured state and to the time index $k$ of the measurement. Thus, $\left\langle\left|u_{k}\right|^{2}\right\rangle_{\tau}$ might be useful when we analyze a large set of experimental data for a specific state at different times.

According to Equation (6) the average transition probability $\left\langle\left|u_{k}\right|^{2}\right\rangle_{\tau}$ reads

$$
\left\langle\left|u_{k}\right|^{2}\right\rangle_{\tau}=P_{N}+\sum_{j, j^{\prime}=1 ; j^{\prime} \neq j}^{N} q_{j}^{*} q_{j^{\prime}} \lambda_{j j^{\prime}}^{k}, \quad P_{N}=\sum_{j=1}^{N}\left|q_{j}\right|^{2} .
$$


The term $P_{N}$ describes the recurrent behavior, which does not depend on time. It is the asymptotic transition probability for $k \rightarrow \infty$

$$
P_{N}=\lim _{k \rightarrow \infty}\left\langle\left|u_{k}\right|^{2}\right\rangle_{\tau}
$$

provided that the energy levels are not degenerated. The case of degenerate energy levels is discussed in the next section. The second term in Equation (7) decays exponentially with time due to $\left|\lambda_{j j^{\prime}}\right|<1$, and only this term describes a change of the transition probability during the evolution of the quantum system. This result provides a separation of the diagonal elements of the TCM into a static recurrent term $P_{N}$ and a dynamic term that decays quickly.

The recurrent term $P_{N}$ provides important information regarding the properties of the quantum system. Since $\left|q_{j}\right|^{2}=\left|\left\langle\Psi \mid E_{j}\right\rangle\right|^{2}\left|\left\langle\Psi_{0} \mid E_{j}\right\rangle\right|^{2}$ is a product of the overlaps between the energy eigenstate $\left|E_{j}\right\rangle$ with the initial state and with the measured state, it is a measure of how much this energy eigenstate contributes to the transition $\left|\Psi_{0}\right\rangle \rightarrow|\Psi\rangle$ during the unitary evolution. This can be used, for instance, to describe localization with the asymptotic behavior of the return probability to the initial state $\left|\Psi_{0}\right\rangle \rightarrow\left|\Psi_{0}\right\rangle$ : With the dimensionality $N$ of the underlying Hilbert space, we get localization when $\lim _{N \rightarrow \infty} P_{N}>$ 0 , whereas the absence of localization is characterized by $\lim _{N \rightarrow \infty} P_{N}=0$ [16]. This can be understood by noting that the normalization of quantum states implies $\sum_{j=1}^{N}\left|\left\langle\Psi_{0} \mid E_{j}\right\rangle\right|^{2}=1$, and that for a localized state, only a few energy eigenstates have nonzero overlaps with $\left|\Psi_{0}\right\rangle$. For a delocalized state, on the other hand, the overlaps are nonzero for a number of order $N$ of energy eigenstates. A special case is when all these overlaps are equal. In that case we have $\left|\left\langle\Psi_{0} \mid E_{j}\right\rangle\right|^{2}=1 / N$ due to the normalization, which implies $P_{N}=1 / N$. Anderson localization is associated with a random Hamiltonian [21]. According to the above described picture, we can also consider Hilbert-space localization for a deterministic Hamiltonian, which depends strongly on the initial state. For an energy eigenstate, the system will always remain in the latter under unitary evolution. More generally, if the initial state is a superposition of $m$ energy eigenstates, the system will always remain inside the $m$-dimensional Hilbert space, spanned by these energy eigenstates. In the case, where the initial state is the eigenstate of $H_{0}$ of the Hamiltonian $H=H_{0}+\eta H_{1}$. $\eta$ is a small parameter. $\eta H_{1}$ is a small perturbation. In that case, it is possible that this perturbation provides an exponentially decaying evolution away the initial state. This would be considered as exponential Hilbert-space localization.

In the subsequent discussion we focus on the diagonal elements of the TCM, since the off-diagonal TCM elements decay exponentially with $\left|k-k^{\prime}\right|$ according to Equation (6).

\subsection{Effect of Spectral Degeneracies}

Assuming that there is a spectral degeneracy $E_{1}=E_{2}$, we have $\lambda_{12}=\lambda_{21}=1$, and the diagonal TCM elements in Equation (7) read in this case

$$
\left\langle\left|u_{k}\right|^{2}\right\rangle_{\tau}=\sum_{j, j^{\prime}=1}^{N} q_{j}^{*} q_{j^{\prime}} \lambda_{j j^{\prime}}^{k}=P_{N}+q_{1}^{*} q_{2}+q_{2}^{*} q_{1}+\sum_{j, j^{\prime}=1 ; j^{\prime} \neq j ;\left(j, j^{\prime}\right) \neq(1,2),(2,1)}^{N} q_{j}^{*} q_{j^{\prime}} \lambda_{j j^{\prime}}^{k},
$$

such that the recurrent part of the transition probability becomes

$$
\lim _{k \rightarrow \infty}\left\langle\left|u_{k}\right|^{2}\right\rangle_{\tau}=P_{N}+q_{1}^{*} q_{2}+q_{2}^{*} q_{1}=\left|q_{1}+q_{2}\right|^{2}+\sum_{j=3}^{N}\left|q_{j}\right|^{2}
$$

Thus, the effect of a spectral degeneracy is a change of the recurrent and the decaying behavior, where the recurrent term changes by $\left|q_{1}+q_{2}\right|^{2}-\left|q_{1}\right|^{2}-\left|q_{2}\right|^{2}$. This means that the diagonal elements of the TCM are very sensitive in terms of spectral degeneracies. 
After applying a discrete Fourier transformation to the decaying part of $\left\langle\left|u_{k}\right|^{2}\right\rangle_{\tau}$, we obtain the function

$$
\begin{aligned}
\tilde{U}_{d}\left(e^{i \omega}\right) & =\sum_{k \geq 1} e^{i \omega k} \sum_{j, j^{\prime}=1 ; k^{\prime} \neq k ;\left(j, j^{\prime}\right) \neq(1,2),(2,1)}^{N} q_{j}^{*} q_{j^{\prime}} \lambda_{j j^{\prime}}^{k} \\
& =\sum_{j, j^{\prime}=1 ; j^{\prime} \neq j ;\left(j, j^{\prime}\right) \neq(1,2),(2,1)}^{N} q_{j}^{*} q_{j^{\prime}} \frac{\lambda_{j j^{\prime}}}{e^{-i \omega}-\lambda_{j j^{\prime}}},
\end{aligned}
$$

which is a function of $\omega$ on the interval $[0,2 \pi)$. In other words, $\tilde{U}_{d}(z)$ is a sum of poles inside the unit circle due to $\left|\lambda_{j j^{\prime}}\right|<1$. The poles $\lambda_{n n^{\prime}}$ and $\lambda_{n^{\prime} n}$ approach the unit circle when we get closer to a degeneracy of $E_{n}$ and $E_{n^{\prime}}$. This should be visible in $\tilde{U}_{d}\left(e^{i \omega}\right)$. The corresponding decay time $T_{d}=-1 / \log \left|\lambda_{n n^{\prime}}\right|$ diverges due to $\left|\lambda_{n n^{\prime}}\right| \sim 1$. Therefore, the decay time $T_{d}$ is a measure of the distance from a spectral degeneracy; it diverges when we approach the degeneracy. In general, we can define

$$
T_{m}=\max _{j, j^{\prime}=1, \ldots, N}-\frac{1}{\log \left|\lambda_{j j^{\prime}}\right|}
$$

as the largest decay time as a measure of level degeneracy.

\section{Example: Bosonic Josephson Junction}

In this section, we study the diagonal TCM elements of a bosonic Josephson junction (BJJ) with $N$ bosons as a closed quantum system. The motivation for choosing this example is at least threefold: The model is (i) simple enough but not trivial, with interesting features based on tunneling and boson-boson interaction; (ii) it can be solved exactly; and (iii) it has been realized experimentally $[22,23]$ with applications for commercial quantum computers [24]. The BJJ consists of two identical wells filled with interacting bosons and a tunneling junction between them. More formally, it is defined by the Bose-Hubbard Hamiltonian [25]:

$$
H=-\frac{J}{2}\left(a_{l}^{\dagger} a_{r}+a_{r}^{\dagger} a_{l}\right)+U\left(n_{l}^{2}+n_{r}^{2}\right), \quad n_{l, r}=a_{l, r}^{\dagger} a_{l, r},
$$

where $a_{l, r}^{+}\left(a_{l, r}\right)$ are the creation (annihilation) operators in the left and right wells, respectively. The first term of $H$ describes tunneling of atoms between the wells, and for $U>0$ the second term represents a repulsive particle-particle interaction that favors energetically a symmetric distribution of bosons in the double well. Without tunneling (i.e., for $J=0$ ) there are two-fold degenerate energy levels $E_{k}=U\left[(N-k)^{2}+k^{2}\right] / 2$ with eigenstates that are superpositions of the product Fock state $|k, N-k\rangle(\equiv|k\rangle \otimes|N-k\rangle)$ and its mirror image $|N-k, k\rangle$. This two-fold degeneracy is similar to the two-fold degeneracy of the Ising model, mentioned in the Introduction. In contrast to the $Z_{2}$ symmetry of the Ising model, the BJJ Hamiltonian has mirror-type symmetry due to the double well structure. Individual tunneling of bosons between the wells plays the role of the symmetry-breaking term, similarly to a local spin flip in the Ising model. Thus, an arbitrarily small tunneling parameter $J$ will lift the two-fold degeneracy of the Fock states. A difference between the Ising model and the BJJ is that without tunneling in the latter, all energy levels are two-fold degenerate. Therefore, the initial state can be prepared in any of these energy levels to follow the evolution due to tunneling in the vicinity of degenerate levels. This is important because the degenerate ground state may not be reached due to the energy conservation in the closed quantum system.

For the following we use $\left|\Psi_{0}\right\rangle=|0, N\rangle$ as the initial state and $|\Psi\rangle=|N, 0\rangle$ as the measured state. Then we define the return probability $(\mathrm{RP})$ and the transition probability (TP) as

$$
\left|u_{r, k}\right|^{2}=\left|\left\langle 0, N\left|e^{-i H t_{k}}\right| 0, N\right\rangle\right|^{2}, \quad\left|u_{t, k}\right|^{2}=\left|\left\langle N, 0\left|e^{-i H t_{k}}\right| 0, N\right\rangle\right|^{2} .
$$


Since both states $\left|\Psi_{0}\right\rangle,|\Psi\rangle$ are eigenstates of $H$ in the absence of tunneling $(J=0)$, we get

$$
\left|u_{r, k}\right|^{2}=1, \quad\left|u_{t, k}\right|^{2}=0
$$

for any $k$ due to orthogonality. This reflects the fact that this pair of states breaks the mirror symmetry of the double well. The opposite extreme is the BJJ without boson-boson interaction $(U=0)$, which is more complex and will be discussed in the next subsection. As we will see, this case can be described by simple functions for $\left|u_{r, k}\right|^{2},\left|u_{t, k}\right|^{2}$. For the interplay of tunneling and boson-boson interaction $(J, U \neq 0)$, the behavior is more complex and we rely on the time averaged expressions $\left\langle\left|u_{r, k}\right|^{2}\right\rangle_{\tau},\left\langle\left|u_{t, k}\right|^{2}\right\rangle_{\tau}$ with $\tau_{k}=\bar{\tau}+\tau_{k}^{\prime}$ and an exponential distribution for $\tau_{k}^{\prime}$ :

$$
\langle\ldots\rangle_{\tau}=\int_{0}^{\infty} \cdots \prod_{n \geq 1} e^{-\tau_{n}^{\prime}} d \tau_{n}^{\prime},
$$

where the time is measured in units of $\hbar / J$.

\subsection{Non-Interacting Bosons}

For $U=0$ the spectrum of $H$ consists of equidistant energy levels $E_{j}=-J(N / 2-j)$ $(j=0,1, \ldots, N)$ and eigenstates

$$
\left|E_{j}\right\rangle=\frac{2^{-N / 2}}{\sqrt{j !(N-j) !}}\left(a_{l}^{+}+a_{r}^{\dagger}\right)^{j}\left(a_{l}^{+}-a_{r}^{\dagger}\right)^{N-j}|0,0\rangle .
$$

Then the RP and the TP at time $t_{k}$ for $N$ bosons read

$$
\begin{gathered}
\left|u_{r, k}\right|^{2}=\left|\left\langle 0, N\left|e^{-i H t_{k}}\right| 0, N\right\rangle\right|^{2}=\left|\cos ^{N}\left(J t_{k} / 2\right)\right|^{2}, \\
\left|u_{t, k}\right|^{2}=\left|\left\langle N, 0\left|e^{-i H t_{k}}\right| 0, N\right\rangle\right|^{2}=\left|\sin ^{N}\left(J t_{k} / 2\right)\right|^{2} .
\end{gathered}
$$

For the average TP we get $t_{k}=k \bar{\tau}+\tau_{1}^{\prime}+\cdots+\tau_{k}^{\prime}$ and the exponential distribution of Equation (16):

$$
\begin{gathered}
\left\langle\left|u_{t, k}\right|^{2}\right\rangle_{\tau}=2^{-2 N} \sum_{l, l^{\prime}=0}^{N}\left(\begin{array}{c}
N \\
l
\end{array}\right)\left(\begin{array}{c}
N \\
l^{\prime}
\end{array}\right)(-1)^{l+l^{\prime}}\left[\frac{e^{-i J \bar{\tau}\left(l-l^{\prime}\right)}}{1-i J\left(l-l^{\prime}\right)}\right]^{k} \\
=2^{-2 N} \sum_{l=0}^{N}\left(\begin{array}{c}
N \\
l
\end{array}\right)^{2}+2^{-2 N} \sum_{l, l^{\prime}=0 ; l^{\prime} \neq l}^{N}\left(\begin{array}{c}
N \\
l
\end{array}\right)\left(\begin{array}{l}
N \\
l^{\prime}
\end{array}\right)(-1)^{l+l^{\prime}}\left[\frac{e^{-i j \bar{\tau}\left(l-l^{\prime}\right)}}{1-i J\left(l-l^{\prime}\right)}\right]^{k},
\end{gathered}
$$

whereas the average RP is the same without the factor $(-1)^{l+l^{\prime}}$. From these results we get for the asymptotic value at $k \sim \infty$ for both probabilities

$$
P_{N} \sim\left\langle\left|u_{r, k}\right|^{2}\right\rangle_{\tau} \sim\left\langle\left|u_{t, k}\right|^{2}\right\rangle_{\tau} \sim 2^{-2 N} \sum_{l=0}^{N}\left(\begin{array}{c}
N \\
l
\end{array}\right)^{2}
$$

which reflects the mirror symmetry of the BJJ.

\subsection{Interacting Bosons}

Exact solutions exist for this model also for $J, U \neq 0$, but in contrast to the noninteracting case they are complex and difficult to present in general. For instance, the resolvent is a meromorphic function with polynomials of orders $N$ and $N+1[16,26]$. Therefore, we only plot the results for the RP and the TP and their averaged counterparts here. For transparency, we chose for all subsequent plots $N=20$ bosons.

First, the evolutions of the RP and the TP are presented in Figure 1 for two values $(u=1,2)$ of the interaction parameter $u=N U / J$. This clearly reveals that the RP dominates over the TP for increasing $u$, as we expected from the results of the two limits 
$J=0$ and $U=0$. It is interesting to note that in a mean-field (classical) approximation of the BJJ there is a sharp phase transition in terms of the interaction parameter, where the mean-field TP is completely suppressed when $u \geq u_{c}=2$ [27]. The strong interaction phase is also called the self-trapping phase. The analogue of the latter in the quantum BJJ is Hilbert-space localization, reflected by the scaling behavior of the inverse participation ratio [16]. This also indicates the existence of a critical $u_{c}$.
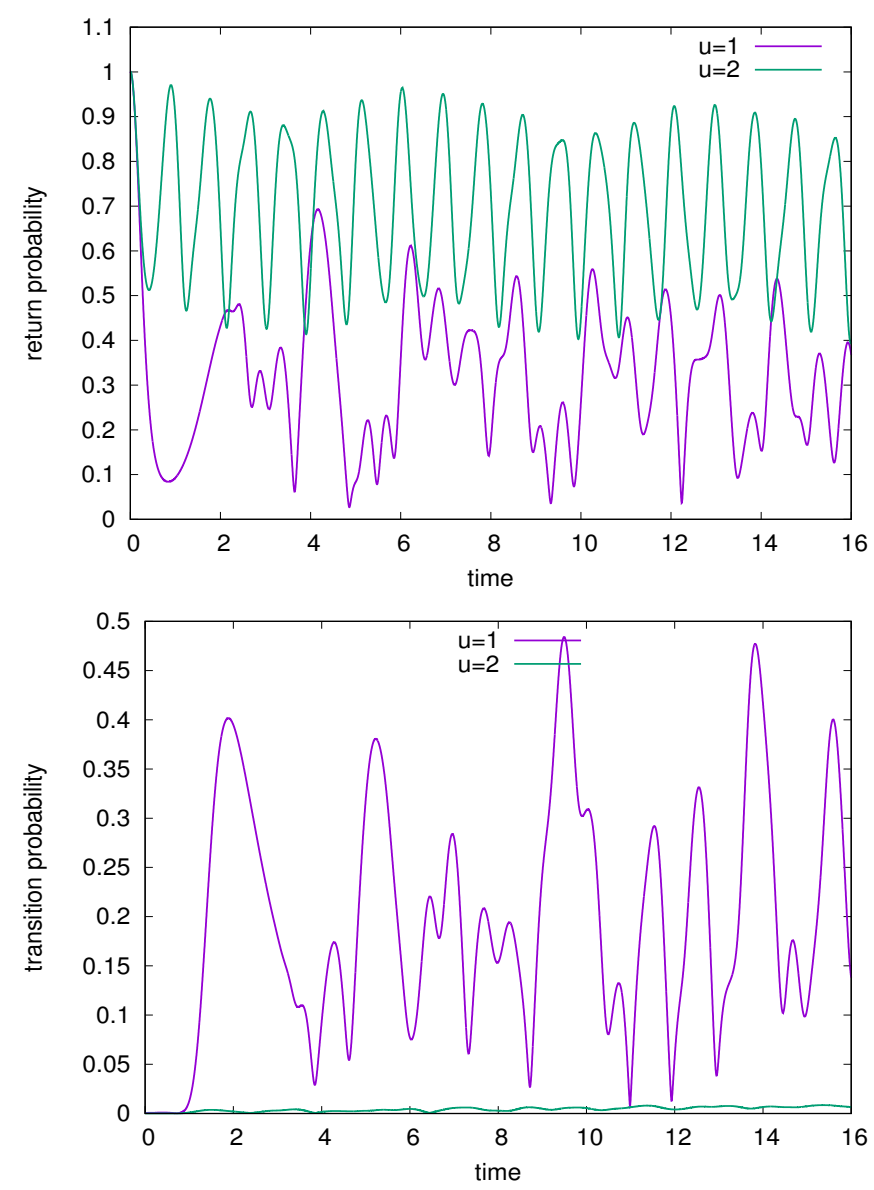

Figure 1. Signatures of a qualitative change in the evolution of a bosonic Josephson junction upon increasing interaction strength $u$. The plots represent the dynamics of 20 bosons for $u=1$, where the top panel gives the return probability $\left|u_{r, k}\right|^{2}$ and the bottom panel the transition probability $\left|u_{t, k}\right|^{2}$.

In Figure 2, the effect of time averaging on $\left|u_{r, k}\right|^{2}$ and $\left|u_{t, k}\right|^{2}$ for $u=1$ is visualized. It reflects the smoothing of the strongly fluctuating dynamics with a recurrent and a decaying contribution according to Equations (7) and (9). It is obvious that the separation of the recurrent and the decaying behavior is not feasible without time averaging.

The existence of a critical interaction strength $u_{c} \approx 1.89 \ldots$ is demonstrated in Figure 3, where the $\left\langle\left|u_{r, k}\right|^{2}\right\rangle_{\tau}$ jumps upon increasing $u$ at $u_{c}$. Moreover, $\left\langle\left|u_{t, k}\right|^{2}\right\rangle_{\tau}$ develops a characteristic peak at $u_{c}$. This behavior reflects the appearance of nearly degenerate energy levels, as described in Section 2.2.

Finally, in Figure 4 the change of the time scales for the decay of the average TP $\left\langle\left|u_{t, k}\right|^{2}\right\rangle_{\tau}$ is visualized for $u=1.7, \ldots, 2.2$. The decay is reduced by increasing interaction strength $u$. This reflects the fact that the splitting of the energy levels is reduced by the interaction, as we expected. 

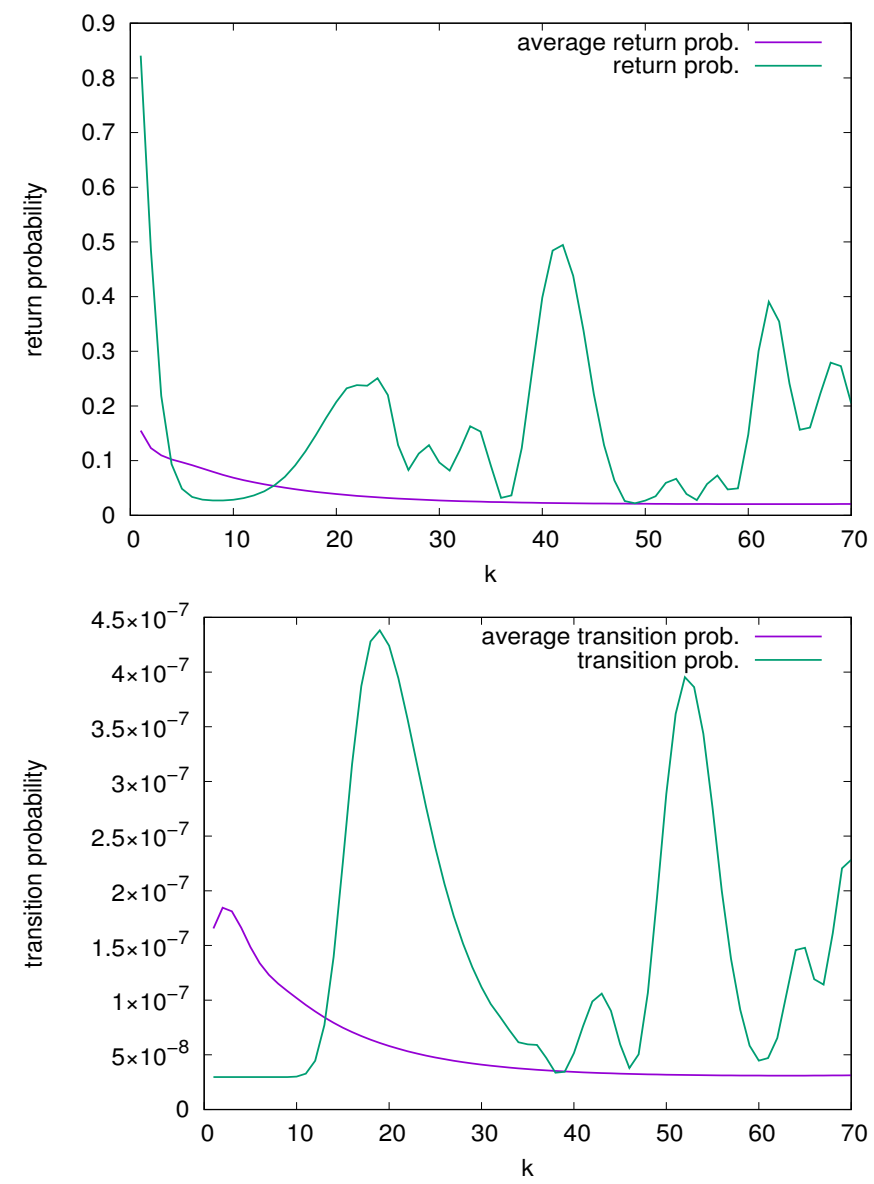

Figure 2. A comparison of the return probability $\left|u_{r, k}\right|^{2}$ and the average return probability $\left\langle\left|u_{r, k}\right|^{2}\right\rangle_{\tau}$ (top panel) and of the corresponding transition probabilities (bottom panel). The average was taken with respect to the exponential distribution of Equation (16). The interaction parameter is $u=1$, and $\bar{\tau}=1 / 10$.

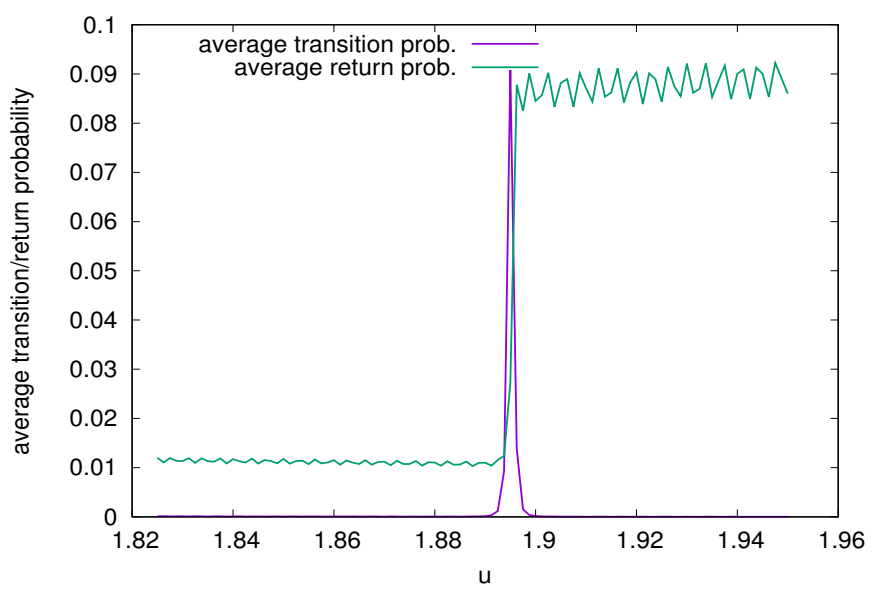

Figure 3. The critical regime of the Hilbert-space localization with $u_{c} \approx 1.89$ is visualized with $\left\langle\left|u_{r, k}\right|^{2}\right\rangle_{\tau}$ and $\left\langle\left|u_{t, k}\right|^{2}\right\rangle_{\tau}$ at $k=70$. 


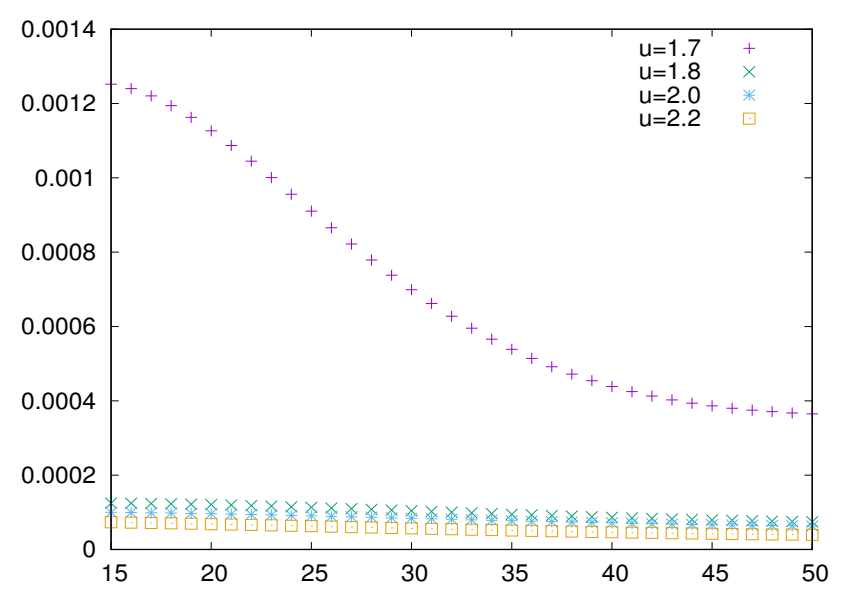

Figure 4. Decay of $\left\langle\left|u_{t, k}\right|^{2}\right\rangle_{\tau}$ for different interaction parameters $u=1.7, \ldots, 2.2$.

\section{Discussion and Conclusions}

Our analysis of the quantum unitary evolution was strictly focused on the result of a single measurement in each of many identical experiments, which were subject of a unitary evolution. Averaging with respect to the statistical outcome due to measurements at randomly distributed times led to the TCM. We focused on the diagonal TCM elements to study the evolution of the quantum system. The analysis of the off-diagonal TCM elements was the subject of a previous work [15]. Similarly to the off-diagonal TCM elements, the diagonal TCM elements revealed separation of the evolution into a static recurrent part and a dynamic decaying part. We found that the decay rate of the latter is related to the spacing between energy levels, which diverges when the spacing vanishes near a degeneracy. Thus, the decay rate is a quantity that can be used to detect symmetry changes or the appearance of spontaneous symmetry breaking. This was observed in the example of the BJJ: In this model the energy levels were two-fold degenerate in the limit $u \rightarrow \infty$. This is reflected in Figure 4, where the decay decreases with increasing $u$.

Another interesting aspect of the BJJ is the transition to Hilbert-space localization [16]. This transition was also detected with the help of the average RP and average TP in Figure 3, where the average RP experiences a jump to a higher value for $u>u_{c}$. On the other hand, the average TP has only a sharp peak near $u_{c}$ but has the same value away from $u_{c}$.

We can conclude that time averaging over an ensemble of measurements is crucial for extracting the (static) recurrent behavior and the (dynamic) decaying behavior. This can be formulated in terms of the TCM. The separation of static and dynamic behavior is generic for the unitary quantum evolution. The TCM provides a tool to analyze properties of the quantum system, which are associated with spectral degeneracy in the case of phase transitions. It can be applied to theoretical calculations and to experimental data, collected from many experiments in identical quantum systems. We have demonstrated in the case of the BJJ that the TCM approach delivers interesting generic information. The BJJ can be considered as a building block of the Bose-Hubbard model on a lattice. Therefore, the TCM approach should be applicable to more complex quantum models, including bosonic and fermionic Hubbard models and quantum spin systems.

Funding: This work was supported by the Julian Schwinger Foundation.

Acknowledgments: I am grateful to Eli Barkai for interesting discussions regarding the Hilbert-space fragmentation.

Conflicts of Interest: The author declares no conflict of interest.

\section{References}

1. Wigner, E.P. Characteristic Vectors of Bordered Matrices With Infinite Dimensions. Ann. Math. 1955, 62, 548-564. [CrossRef]

2. Porter, C.E.; Thomas, R.G. Fluctuations of Nuclear Reaction Widths. Phys. Rev. 1956, 104, 483-491. [CrossRef] 
3. Dyson, F.J. Statistical Theory of the Energy Levels of Complex Systems. I. J. Math. Phys. 1962, 3, 140-156. [CrossRef]

4. Porter, C.E. Book Review: Statistical theories of spectra: fluctuations. C.E. PORTER (Academic Press, New York, $1965 . x v-576$ p. 5.95 paper, 9.50 cloth). Nucl. Phys. 1966, 78, 696-696. [CrossRef]

5. Wigner, E.P. Random Matrices in Physics. SIAM Rev. 1967, 9, 1-23. [CrossRef]

6. Mehta, M. Random Matrices; Number v. 142 in Pure and Applied Mathematics; Academic Press: Cambridge, MA, USA, 2004.

7. Beenakker, C.W.J. Random-matrix theory of quantum transport. Rev. Mod. Phys. 1997, 69, 731-808. [CrossRef]

8. Dyson, F.J. Statistical Theory of the Energy Levels of Complex Systems. II. J. Math. Phys. 1962, 3, 157-165. doi: 10.1063/ 1.1703774. [CrossRef]

9. Dyson, F.J. Statistical Theory of the Energy Levels of Complex Systems. III. J. Math. Phys. 1962, 3, 166-175. . [CrossRef]

10. van Enk, S.J.; Beenakker, C.W.J. Measuring $\operatorname{Tr} \rho^{n}$ on Single Copies of $\rho$ Using Random Measurements. Phys. Rev. Lett. 2012, 108, 110503. [CrossRef]

11. Elben, A.; Vermersch, B.; Dalmonte, M.; Cirac, J.I.; Zoller, P. Rényi Entropies from Random Quenches in Atomic Hubbard and Spin Models. Phys. Rev. Lett. 2018, 120, 050406. [CrossRef]

12. Vermersch, B.; Elben, A.; Dalmonte, M.; Cirac, J.I.; Zoller, P. Unitary n-designs via random quenches in atomic Hubbard and spin models: Application to the measurement of Rényi entropies. Phys. Rev. A 2018, 97, 023604. [CrossRef]

13. Li, Y.; Chen, X.; Fisher, M.P.A. Measurement-driven entanglement transition in hybrid quantum circuits. Phys. Rev. B 2019, 100, 134306. [CrossRef]

14. Skinner, B.; Ruhman, J.; Nahum, A. Measurement-Induced Phase Transitions in the Dynamics of Entanglement. Phys. Rev. X 2019, 9, 031009. [CrossRef]

15. Ziegler, K. Quantized dynamics in closed quantum systems. J. Phys. Math. Theor. 2021, 54, 205303. [CrossRef]

16. Cohen, D.; Yukalov, V.I.; Ziegler, K. Hilbert-space localization in closed quantum systems. Phys. Rev. A 2016, $93,042101$. [CrossRef]

17. Moudgalya, S.; Motrunich, O.I. Hilbert Space Fragmentation and Commutant Algebras. arXiv 2021, arXiv:2108.10324.

18. Cotler, J.S.; Gur-Ari, G.; Hanada, M.; Polchinski, J.; Saad, P.; Shenker, S.H.; Stanford, D.; Streicher, A.; Tezuka, M. Black holes and random matrices. J. High Energy Phys. 2017, 2017, 118. [CrossRef]

19. Kos, P.; Ljubotina, M.; Prosen, T.C.V. Many-Body Quantum Chaos: Analytic Connection to Random Matrix Theory. Phys. Rev. X 2018, 8, 021062. [CrossRef]

20. Chan, A.; De Luca, A.; Chalker, J.T. Solution of a Minimal Model for Many-Body Quantum Chaos. Phys. Rev. X 2018, 8, 041019. [CrossRef]

21. Anderson, P.W. Absence of Diffusion in Certain Random Lattices. Phys. Rev. 1958, 109, 1492-1505. [CrossRef]

22. Bar-Gill, N.; Kurizki, G.; Oberthaler, M.; Davidson, N. Dynamic control and probing of many-body decoherence in double-well Bose-Einstein condensates. Phys. Rev. A 2009, 80, 053613. [CrossRef]

23. Juliá-Díaz, B.; Zibold, T.; Oberthaler, M.K.; Melé-Messeguer, M.; Martorell, J.; Polls, A. Dynamic generation of spin-squeezed states in bosonic Josephson junctions. Phys. Rev. A 2012, 86, 023615. [CrossRef]

24. Barends, R.; Kelly, J.; Megrant, A.; Sank, D.; Jeffrey, E.; Chen, Y.; Yin, Y.; Chiaro, B.; Mutus, J.; Neill, C.; et al. Coherent Josephson Qubit Suitable for Scalable Quantum Integrated Circuits. Phys. Rev. Lett. 2013, 111, 080502. [CrossRef] [PubMed]

25. Gati, R.; Oberthaler, M.K. A bosonic Josephson junction. J. Phys. At. Mol. Opt. Phys. 2007, 40, R61-R89. [CrossRef]

26. Ziegler, K. Dynamical creation of entangled bosonic states in a double well. J. Phys. At. Mol. Opt. Phys. 2011, 44, 145302. [CrossRef]

27. Milburn, G.J.; Corney, J.; Wright, E.M.; Walls, D.F. Quantum dynamics of an atomic Bose-Einstein condensate in a double-well potential. Phys. Rev. A 1997, 55, 4318-4324. [CrossRef] 